\title{
Calixarene-encapsulated nanoparticles: self-assembly into functional nanomaterials $\dagger$
}

\author{
Alexander Wei* \\ Received (in Cambridge, UK) 7th November 2005, Accepted 7th December 2005 \\ First published as an Advance Article on the web 25th January 2006 \\ DOI: $10.1039 / b 515806 k$
}

Calixarenes are excellent surfactants for enhancing the dispersion and self-assembly of metal nanoparticles into well-defined structures, particularly those with unit length scales in the $10-100 \mathrm{~nm}$ size range. Particles within these ensembles are strongly coupled, giving rise to unique collective optical or magnetic properties. The self-assembled nanostructures described in this feature article include 2D arrays of colloidal Au nanoparticles with size-dependent plasmonic responses, and sub-100 nm Co nanoparticle rings with chiral magnetic states. These nanoparticle assemblies may be further developed for applications in chemical sensing based on surfaceenhanced Raman scattering (SERS) and as binary elements for nonvolatile memory, respectively.

\section{Introduction}

Scientists have had a long-standing fascination for selfassembled systems, and the opportunities presented for the spontaneous creation of functional materials and devices from relatively simple building blocks. At the molecular level, chemists have become skilled at employing various supramolecular interactions to produce multicomponent assemblies with a sophisticated level of structural complexity and exciting technological potential. ${ }^{1}$ As self-assembly progresses toward nanoscale dimensions, forces of a different nature become significant: attractive long-range van der Waals (vdW) interactions, electrostatic double-layer forces, and repulsive

Department of Chemistry and the Birck Nanotechnology Center, Purdue University, 560 Oval Drive, West Lafayette, IN, USA.

E-mail:alexwei@purdue.edu; Fax: +1 765494 0239; Tel: +1 7654945257

$\uparrow$ Dedicated to the memory of Professor Ian P. Rothwell.

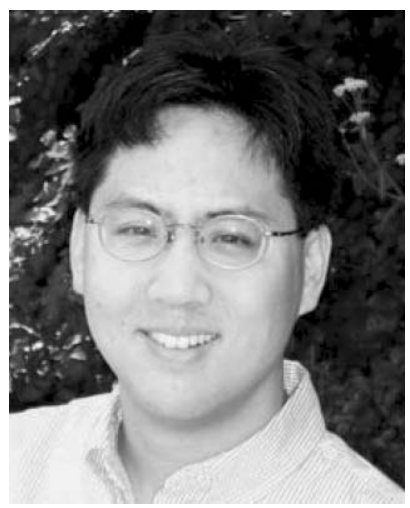

Alex Wei
Alex Wei received his $B S$ degree with honors in Chemistry from Caltech in 1989, where he conducted research with J. D. Roberts in nitrogen-15 NMR spectroscopy. He obtained his PhD from Harvard University in 1995 on the synthesis of C-glycosides under the guidance of Y. Kishi, and as a Fulbright scholar carried out postdoctoral research at Université Louis Pasteur in Strasbourg with J.-M. Lehn. He began his independent career at Purdue University in 1997, where he is presently an Associate Professor in the Department of Chemistry and the Birck Center for Nanotechnology. His research interests include nanoscale self-assembly, supramolecular surface science, cellsurface carbohydrates, and their applications to biological systems. short-range interactions to buffer particles against irreversible aggregation or adsorption to surfaces. ${ }^{2}$ Rational control over these forces becomes increasingly important for the selfassembly of novel nanoscale architectures with predesignated functions.

In this feature article we examine calixarenes for enhancing the dispersion and self-assembly of metal nanoparticles, as well as fundamental and applied studies of their collective physical properties. The macrocyclic headgroup and amphiphilic nature of calixarenes are ideally suited for nanoparticle encapsulation and dispersion, particularly those with diameters $(d)$ on the order of 10-100 $\mathrm{nm}$. Although such nanomaterials are widely available (often from commercial sources) and display useful optical or magnetic properties, they remain relatively underexploited due to the refractory nature of colloidal dispersion forces. We have found that calixarene-based surfactants can lower this working barrier substantially; for example, gold particles as large as $170 \mathrm{~nm}$ can be induced to self-assemble on air-water interfaces when encapsulated by calixarenes.

Our choice of calixarenes have been centered on the resorcinarenes (see Fig. 1), first introduced by Högberg ${ }^{3}$ and developed for supramolecular applications by Cram, ${ }^{4}$

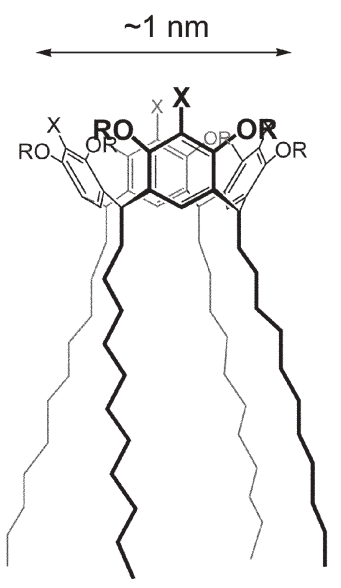

C11 Resorcinarene (variable $\mathrm{R}$ and $\mathrm{X}$ ) 
Aoyama, ${ }^{5}$ Reinhoudt, ${ }^{6}$ and many others. Resorcinarenes can be synthesized from resorcinol and simple aldehydes in a single step and high yield, ${ }^{5}$ and provide a versatile framework for systematic modifications to the headgroup or tailgroups. ${ }^{6}$ However, the resorcinarenes' dispersant properties are not necessarily unique: other calixarenes, cyclodextrins, ${ }^{7}$ and nonplanar macrocycles with dendritic features may also be developed for nanoparticle encapsulation and self-assembly.

In order to place the title subject within the context of related research efforts, we begin with a general overview of nanoparticle self-assembly, followed by a brief discussion on the role of colloidal forces in surfactant design.

\section{Nanoparticle self-assembly}

Self-assembly processes have tremendous appeal for organizing nanosized particles into materials with well-defined structure. Self-assembly can generate periodic structures well below the resolution limits of conventional lithography, and in a scalable fashion with low overhead cost. Moreover, nanoscale ensembles often exhibit unique materials properties as a result of electronic or electromagnetic coupling between unit particles. These collective states differ from those of either individual nanoparticles or the bulk solid, and can be adjusted by changes in physical parameters such as particle size, interparticle spacing, and higher-order lattice structure. ${ }^{8}$ Such tunable properties are the quintessence of nanoscale science and technology, and have given rise to the popular concept of nanoparticles as "artificial atoms", and their superlattices as "quantum dot solids," or more recently as "metamaterials." For instance, 2D arrays of alkanethiolcoated Ag nanoparticles have been shown to undergo an insulator-to-metal transition when subjected to lateral compression, ${ }^{10}$ and arrays of superparamagnetic Co nanoparticles are capable of spin-dependent electronic transport upon a reduction in interparticle spacing. ${ }^{11}$

The potential of self-assembly to produce nanomaterials with tunable properties or device-like function has sparked a global research effort to develop "bottom-up" approaches using an expanded set of nanoscale building blocks. Indeed, the majority of $2 \mathrm{D}$ and $3 \mathrm{D}$ nanoscale assemblies have been comprised of spherical nanoparticles with $d<10 \mathrm{~nm}$, and much of the literature on this topic has been compiled in several reviews. ${ }^{8,12}$ More recently, anisotropic particles such as nanorods have been organized into monolayers or liquid crystalline phases, ${ }^{13}$ and fluid-directed assembly has been used to deposit nanowires as crossbars onto electrical contact pads. ${ }^{14}$ "Programmed" nanoparticle self-assembly mediated by surfactants with molecular recognition elements has also been demonstrated, ${ }^{7,15}$ and has been used to control 2D organization in certain instances. ${ }^{16}$ Spherical nanoparticles can also self-assemble into architectures of greater complexity; for example, mixtures of two different particle types can organize into binary superlattices, whose lattice structures are determined by relative particle size and stoichiometry. ${ }^{17}$

Methods for nanoparticle self-assembly vary considerably, depending on the nature of the particles used and the media in which they are dispersed. For nanoparticles with $d<10 \mathrm{~nm}$, $\mathrm{vdW}$ forces are typically at or below thermal energies $\left(k_{\mathrm{B}} T\right)$, such that self-assembly can be driven by interactions at molecular length scales (e.g., surfactant chain interdigitation and hydrogen bonding). ${ }^{15,18,19}$ Stable dispersions of nanoparticles can self-assemble into 2D arrays onto wettable surfaces by simple dropcasting techniques, ${ }^{11,19,20}$ although long-range order is critically dependent on the solvent evaporation rate. ${ }^{21}$ Nanoparticles can also be deposited onto air-water interfaces and compressed into close-packed 2D arrays in a LangmuirBlodgett trough, ${ }^{10,18,22}$ or organized into 3D superlattices by molecular crystal growth techniques in organic media. ${ }^{23}$ Liquid interfaces are especially useful for tuning particle interactions, and permit self-assembled films to be transferred onto solid surfaces with a high degree of fidelity. ${ }^{24}$

Long-range vdW attractions become difficult to control when working with metal or inorganic nanoparticles whose dimensions extend well beyond molecular length scales, i.e. 10$100 \mathrm{~nm}$. The interaction potentials increase rapidly with particle size, and exceed the forces typically employed in supramolecular assembly. However, mid-nanometer sized particles also exhibit useful size-dependent characteristics, such as the surface plasmon modes in gold and silver nanoparticles $^{25}$ or the onset of ferromagnetic behavior in magnetic nanoparticles. ${ }^{26}$ The dispersion and self-assembly of nanoparticles in the $10-100 \mathrm{~nm}$ size range thus constitutes a critical enabling step in the technological development of these functional nanomaterials.

\section{Surfactant design considerations}

To produce stable nanoparticle dispersions in nonaqueous media, one must employ surfactants which provide both miscibility and sufficient steric repulsion for countering longrange vdW attraction. This repulsion is considered to be largely entropic in nature and highly dependent on chain mobility: as two surfaces approach, the compression of the surfactant layers is accompanied by an increasing loss of configurational entropy, producing a secondary minimum (see Fig. 2). In the case of small $(d<10 \mathrm{~nm})$ nanoparticles, singlechain surfactants such as alkanethiols provide sufficient steric repulsion to enable self-assembly with thermodynamic control, as the particle curvature is high enough to allow for significant chain mobility (see Fig. 3(a)).

Unfortunately, this situation does not hold for particle sizes significantly larger than $10 \mathrm{~nm}$. In addition to stronger longrange vdW attractions, metal nanoparticles typically have planar facets which encourage surfactants to assemble into close-packed domains, resulting in low chain mobility. Under these conditions short-range steric repulsion is easily overwhelmed by vdW attractive forces, with poor control over dispersion stability and particle aggregation (see Fig. 3(b)).

Entropic steric repulsion can be greatly increased by modulating the density of chains on the nanoparticle surfaces. This concept is well accepted in polymer brush theory and used to describe repulsive interactions between polymer-coated surfaces, and may also be instructive for describing interactions between surfactant-coated nanoparticles. According to the Alexander-DeGennes brush model, ${ }^{27}$ two surfaces with surfactant coatings of thickness $L$ in a good solvent will experience a repulsive pressure $P(\delta)$, where $\delta$ is the separation 


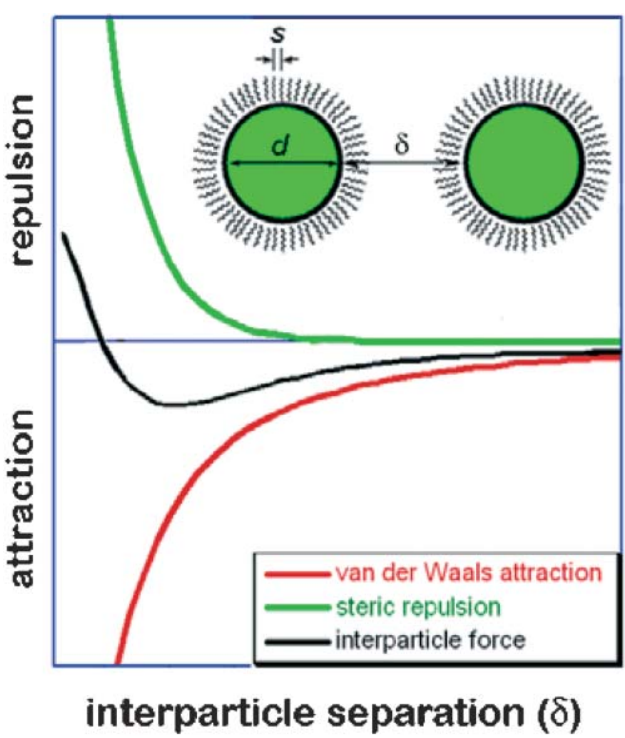

Fig. 2 Description of forces between two particles with diameter $d$, coated with surfactant layers having separation $s$ between chains. Thermodynamic self-assembly can be achieved when long-range vdW attraction (red) is countered by short-range steric repulsion (green).
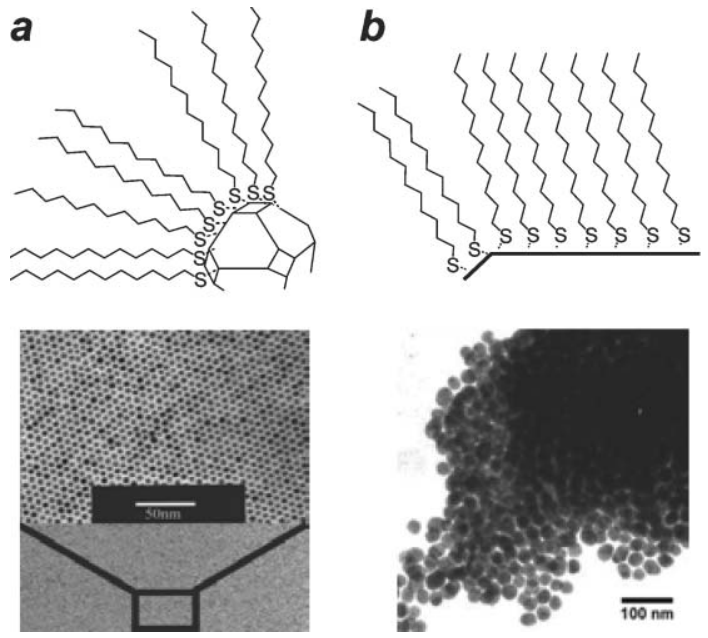

Fig. 3 (a) Single-chain surfactants such as $C_{12}$ thiol retain significant chain mobility when adsorbed onto particles with $d<10 \mathrm{~nm}$. A selfassembled 2D array of $5.5-\mathrm{nm}$ thiol-coated $\mathrm{Au}$ particles is shown below, prepared with slow evaporation of the wetting layer. ${ }^{21}$ (b) $\mathrm{C}_{12}$ thiols form close-packed domains with minimal chain entropy when adsorbed on particles with $d>10 \mathrm{~nm}$. An attempt to assemble 20-nm thiol-coated $\mathrm{Au}$ particles on an aqueous interface produced a disordered aggregate. ${ }^{44}$

or spacing between hard surfaces (see Fig. 2). This pressure is roughly exponential when $\delta<2 L$ (specifically, when $\delta / 2 L$ is between 0.2 to 0.9 ):

$$
P(\delta) \approx \frac{100 k T}{s^{3}} e^{-\pi \delta / L}
$$

where $s$ is the average distance between grafted chains.

The polymer brush model may be extended qualitatively to molecular surfactant layers if they share some of the criteria for 'brush' conditions. Namely, the chains must have a separation $s$ which is significantly smaller than their length, and should have no appreciable self-affinity. However, densely packed monolayers of single-chain surfactants often do not meet these criteria because of their strong tendency to form crystalline domains (see Fig. 3(b)). ${ }^{28}$ The chains' conformational freedom is greatly restricted within the compact monolayer, with site densities nearly equal to their molecular cross section. The surfactant layers thus behave as if exposed to poor solvent conditions, and experience little or no loss of entropy when approached by another surface.

Surfactants based on the resorcinarene framework offer some important advantages over conventional surfactants for enhancing nanoparticle dispersion and self-assembly. Resorcinarenes possess two salient features which contribute to their superior dispersant qualities: (i) large, multivalent headgroups for robust adsorption to the nanoparticle surface, and (ii) several hydrocarbon tails per molecule spaced several angstroms apart (see Fig. 1). The latter ensures a high degree of configurational freedom per chain in the surfactant layer, which translates into effective entropic steric repulsion regardless of the molecular packing density on the nanoparticle surface.

It is very much worth mentioning that others have found calixarenes to be useful molecules on nanoparticle surfaces or for nanosized capsule formation. Atwood and co-workers have provided a number of elegant X-ray crystal structures of resorcinarenes and pyrogallolarenes assembled into closedshell hexamers with inner cavities on the order of $2 \mathrm{~nm},{ }^{29}$ and a tetrahedral cavitand tetra(dithiocarbamate) $\mathrm{Zn}$ coordination complex has been reported by Beer and co-workers. ${ }^{30}$ Nanosized calixarene capsules have also been studied in the solution state by Rebek and Cohen. ${ }^{31}$ With respect to nanoscale surface chemistry, Reinhoudt and co-workers have investigated nanoparticles coated by calixarenes with the headgroups facing outward, ${ }^{32}$ and Richardson and Kaifer have examined the use of calixarenes for templating nanoparticle growth. ${ }^{33}$ At the submicron scale, Ichimura and coworkers have encapsulated colloidal silica particles with resorcinarenes having azobenzenes in their tailgroups, and demonstrated reversible changes in dispersibility based on cistrans photoisomerization. ${ }^{34}$

\section{Encapsulation of gold nanoparticles}

Our initial studies involved the use of $C$-undecylcalix[4]resorcinarene $\left(\mathrm{C}_{11}\right.$ resorcinarene) $\mathbf{1}$ and octamethyl derivative 2 to encapsulate and stabilize $\mathrm{Au}$ nanocrystals dispersed in hydrocarbons, in collaboration with Andres (Dept. of Chemical Engineering, Purdue University). Uncoated nanocrystals were produced as aerosols and captured by passage through a millimolar solution of $\mathbf{1}$ or $\mathbf{2}$ in mesitylene. ${ }^{35}$ The resulting dispersions were indefinitely stable and exhibited a red-purple hue characteristic of the plasmon resonance produced by Au nanoparticles, with optical densities several times greater than those obtained using millimolar solutions of dodecanethiol (see Fig. 4, right). Analysis by transmission electron microscopy (TEM) revealed that the resorcinarene solutions were capable of stabilizing nanoparticles up to $20 \mathrm{~nm}$ in diameter, whereas the corresponding dodecanethiol 


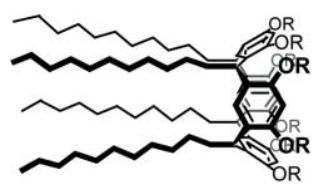

1: $\mathrm{R}=\mathrm{H}$

2: $\mathrm{R}=\mathrm{CH}_{3}$

3: $\mathrm{R}=-\mathrm{CH}_{2}$

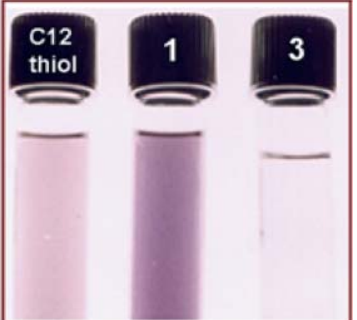

Fig. 4 Left, $\mathrm{C}_{11}$ resorcinarene derivatives 1-3; right, Au nanocrystals encapsulated by $\mathrm{C}_{12}$ thiol, resorcinarene $\mathbf{1}$, and cavitand 3 (control).

$\left(\mathrm{C}_{12}\right.$ thiol $)$ solutions only supported nanoparticles with $d<5 \mathrm{~nm}$ (see Fig. 5).

Resorcinarenes $\mathbf{1}$ and $\mathbf{2}$ were comparable in their efficacies to capture Au nanocrystals, which indicated that encapsulation was mediated by direct chemisorption rather than by formation of a supramolecular, hydrogen-bonded shell. The chemisorption of $\mathbf{2}$ was further supported by spectroscopic studies based on surface-enhanced Raman scattering (SERS), which ruled out the possibility of nonspecific physisorption. The stability of the chemisorbed resorcinarenes was surprising, given the weak affinity between $\mathrm{Au}$ and $\mathrm{O}$. However, the cooperative adsorption of multiple oxygen atoms in $\mathbf{2}$ was evidently sufficient to passivate the metal surface in the absence of other strongly chemisorptive species. In comparison, tetra- $O, O$-methylene derivative (cavitand) 3 was unable to capture nanocrystals in significant quantities, as the bridging methylene units prevented cooperative adsorption of the oxygen lone pairs to the metal surface.

The resorcinarenes' ability to disperse the larger $\mathrm{Au}$ nanocrystals was confirmed by introducing $\mathrm{C}_{12}$ thiol as a competing surfactant. A decrease in optical density was observed over a period of three days until a markedly lighter solution was achieved, similar to the thiol-stabilized nanoparticle solution in Fig. 4 save for a dark precipitate at the bottom of the vial. TEM analysis revealed that only particles with $d<5 \mathrm{~nm}$ remained in the dispersion (see Fig. 5), indicating that the larger particles had precipitated after displacement of the resorcinarene coating by $\mathrm{C}_{12}$ thiol.
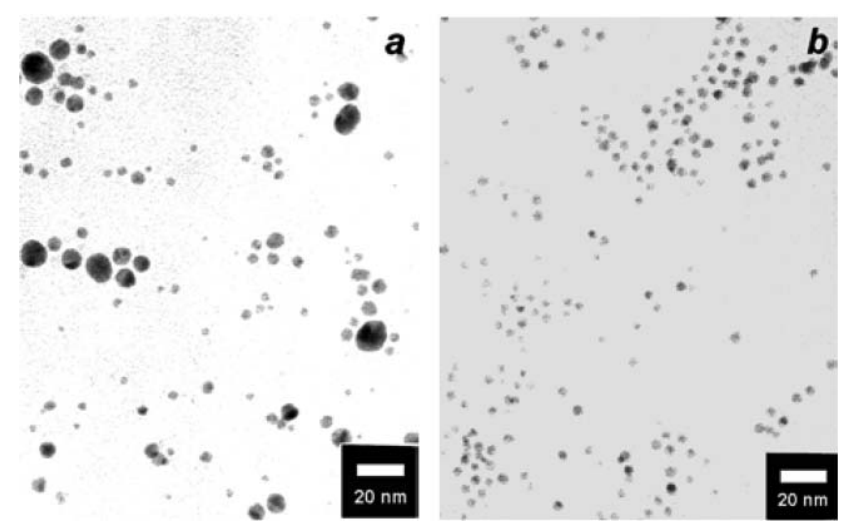

Fig. 5 (a) TEM image (JEOL 2000-FX, $200 \mathrm{keV}$ ) of Au nanocrystals dispersed in mesitylene by $\mathrm{C}_{11}$ resorcinarene octamethyl ether 2. (b) $\mathrm{Au}$ nanocrystals three days after treatment with $\mathrm{C}_{12}$ thiol. ${ }^{35}$

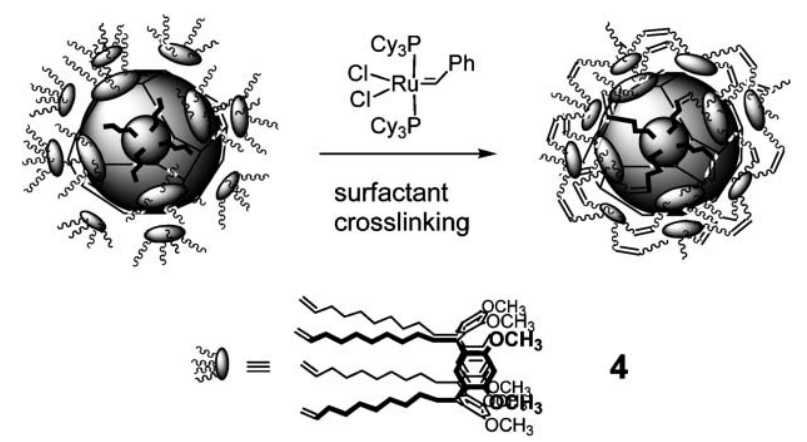

Fig. 6 Encagement of Au nanocrystals in crosslinked surfactant shell of resorcinarene $4^{36}$

The study above confirmed that the $\mathrm{C}_{11}$ resorcinarenes were remarkable as nanoparticle dispersants, but at the same time their adsorption to the metal surface was too labile to be useful for further manipulations. To address this issue, we first considered encaging nanoparticles inside of nondesorptive shells by covalent crosslinking of the resorcinarene monolayer. Tetraene 4 was used to encapsulate Au nanocrystals in the manner described above, followed by treatment with Grubbs' ruthenium carbene catalyst for crosslinking the terminal alkenes by olefin metathesis (see Fig. 6). ${ }^{36}$ This proved to be successful in creating a robust surfactant shell: encaged nanoparticles could be passed through a polystyrene sizeexclusion column without significant degradation, whereas encapsulated nanoparticles which were not subjected to olefin metathesis did not survive chromatography. However, crosslinking of the surfactant chains reduced the dispersibility of the encaged nanoparticles, compromising the processing advantages of the resorcinarene surfactant layer. While changes to the crosslinking procedure have yielded nondesorptive nanoparticle coatings with improved dispersion characteristics, ${ }^{37}$ it was evident that robust nanoparticle encapsulation could be attained more readily via modifications to the macrocyclic headgroup.

Tetraphosphonite and tetrathiol derivatives 5-7 were all found to be highly effective at dispersing Au nanoparticles and were robust to desorption from the metal surface. ${ }^{38,39}$ Moreover, resorcinarene tetrathiols 6 and especially 7 were capable of extracting colloidal Au nanoparticles from aqueous solutions.t. These classic 'Faraday sols' are easily produced in the $10-100 \mathrm{~nm}$ size range with narrow size distributions and are endowed with size-dependent optical properties, but their organization into well-defined ensembles had never been systematically addressed because of difficulties in dispersion control.
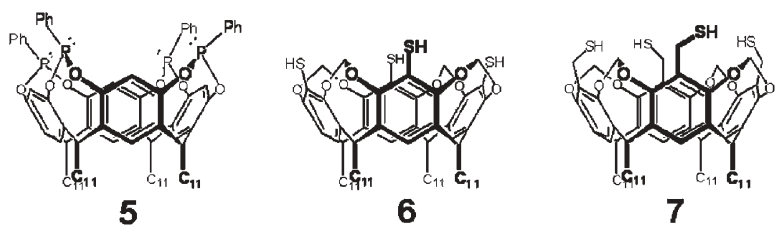

$\$$ Adding trace amounts of a hydrophobic salt (e.g., $n$-Oct ${ }_{4} \mathrm{NBr}$ ) can aid the extraction into organic solvents by exchanging with residual inorganic cations associated with the encapsulated nanoparticles. 


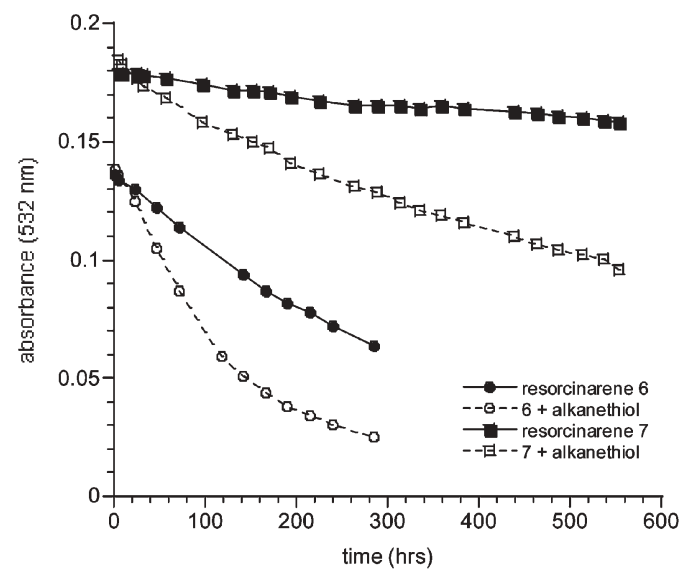

Fig. 7 Dispersion stability of $19-\mathrm{nm}$ Au particles in toluene at $25^{\circ} \mathrm{C}$ encapsulated by resorcinarene tetrathiols 6 and 7, in the absence and presence of alkanethiols (filled and open markers). ${ }^{39}$

Colloidal Au particles as large as $87 \mathrm{~nm}$ could be dispersed in chloroform-THF mixtures by tetrabenzylthiol resorcinarene 7. ${ }^{39}$ The stability of the nanoparticles encapsulated by tetrathiols 6 and 7 was also evaluated against alkanethiolinduced precipitation in organic solvents. Both systems were far more stable than the nanocrystals coated with resorcinarenes 1 or $\mathbf{2}$, but 7 was significantly more robust in its chemisorption than $\mathbf{6}$ and could maintain a long dispersion half-life $\left(t_{1 / 2} \sim 1\right.$ month) in the presence of alkanethiols at ambient temperature (see Fig. 7). X-Ray photoelectron spectroscopy (XPS) of the resorcinarenes on planar $\mathrm{Au}$ substrates revealed that tetraarylthiol $\mathbf{6}$ was attached by essentially a single $\mathrm{Au}-\mathrm{S}$ bond, whereas tetrabenzylthiol 7 was stabilized by at least two Au-S bonds. This indicated that the quality of chemisorption is dictated by the accessibility of the headgroup thiol to the Au surface.

The strength of chemisorption can be further increased by using dithiocarbamate (DTC) units for surface ligation. DTCs are readily formed by condensation of $\mathrm{CS}_{2}$ with alkylamines, in many cases without the need for additional base. ${ }^{40}$ For example, a solution of tetra $(N$-methyl)aminomethyl resorcinarene in aqueous THF was treated with $\mathrm{CS}_{2}$ to form DTC ligand 8, which could then extract $\mathrm{Au}$ nanoparticles into $\mathrm{CH}_{2} \mathrm{Cl}_{2}$ (see Fig. 8). Very importantly, the chemisorption of DTCs on Au can withstand high temperatures and is highly

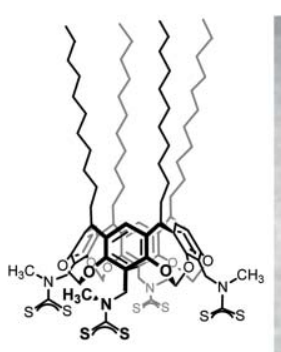

8
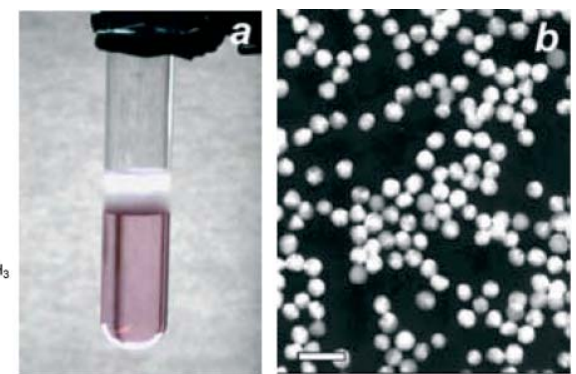

Fig. 8 (a) Extraction of $40-\mathrm{nm}$ Au particles into $\mathrm{CH}_{2} \mathrm{Cl}_{2}$ by resorcinarene tetra-DTC 8, formed by the condensation of $\mathrm{CS}_{2}$ with the corresponding tetraamine. (b) TEM image (Philips EM-400, $80 \mathrm{kV}$ ) of $\mathrm{Au}$ nanoparticles encapsulated by $\mathbf{8}$; scale bar $=100 \mathrm{~nm} .{ }^{40}$ resistant to displacement by thiols, suggesting its utility in applications involving harsh environmental conditions or biological fluids.

\section{Self-organization of gold nanoparticles at aqueous interfaces}

One factor which must be taken into account during the extraction of gold hydrosols is the presence of adsorbed electrolytes such as chloride or citrate, which gives rise to an appreciable electrostatic double layer. This can be controlled by using an ion-exchange resin to minimize the ionic strength of the colloidal suspensions prior to introducing the surfactant. ${ }^{39,41}$ Even so, a sublayer of adsorbed electrolyte remains; if the surfactant is not aggressive enough to completely displace the adsorbed ions, the encapsulated nanoparticles will not be fully extracted to the organic phase but instead be confined to the aqueous interface. $f$

These effects are illustrated in Fig. 9, in which tetrathiol resorcinarenes 6 and 7 and pyridinearene 9 were used to extract $35-\mathrm{nm} \mathrm{Au}$ particles from aqueous solution. ${ }^{42}$ The strongly passivating tetrabenzylthiol 7 could extract nanoparticles to the organic phase even in the presence of competing electrolyte, whereas the weakly adsorbing pyridinearene 9 enabled only a partial extraction to the aqueous interface at minimal ionic strength. Nanoparticles encapsulated by tetraarylthiol 6 were amphipathic under either condition, indicative of strong chemisorption but incomplete passivation of the particle surface.

Confining the resorcinarene-encapsulated nanoparticles to the aqueous interface resulted in a dark blue or metallic film, due to the electromagnetic coupling of densely packed nanoparticles (see next section). These films could be reliably transferred onto glass or quartz substrates by collecting and redepositing the particles onto a fresh air-water interface, followed by the slow withdrawal of a partially immersed

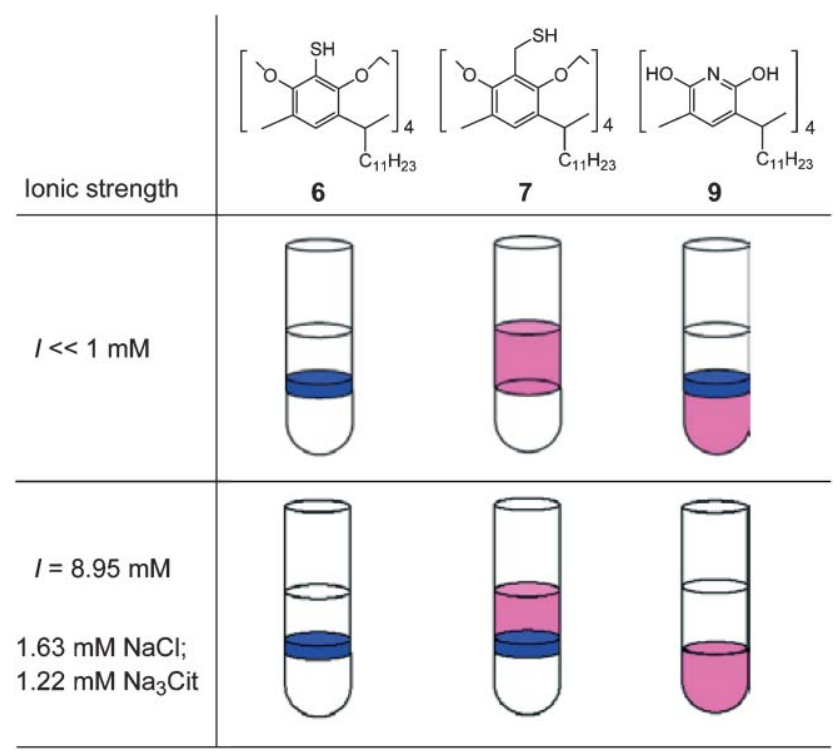

Fig. 9 Illustrations of nanoparticle extractions $(d=35 \mathrm{~nm})$ by resorcinarenes $\mathbf{6}$ and $\mathbf{7}$ and pyridinearene $\mathbf{9}$, from solutions of different ionic strength. ${ }^{42}$ Nanoparticle films at the aqueous interface are dark blue. 


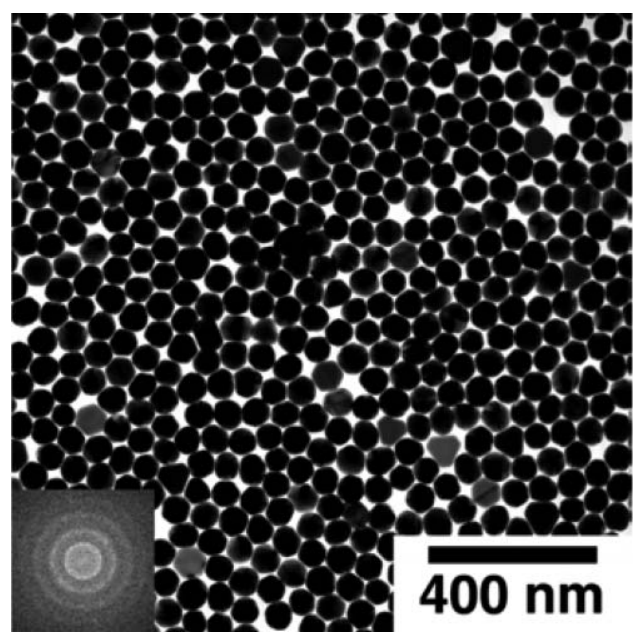

Fig. 10 TEM image (Philips EM-400, $80 \mathrm{kV}$ ) of self-assembled Au nanoparticle array. ${ }^{43} 70-\mathrm{nm} \mathrm{Au}$ particles were encapsulated and extracted to the air-water interface by resorcinarene tetrathiol $\mathbf{6}$. Fourier transform of TEM image (lower left) provides an indication of $2 \mathrm{D}$ order.

substrate. TEM analysis revealed that the nanoparticles were assembled into close-packed arrays of monoparticulate thickness and hexagonal order (see Fig. 10). ${ }^{42,43}$ After a systematic investigation of deposition and film transfer conditions, it was determined that the nanoparticles spontaneously organized into $2 \mathrm{D}$ arrays while suspended at the air-water interface.

To examine the generality of this self-assembly process, $\mathrm{Au}$ nanoparticles ranging from 16 to $170 \mathrm{~nm}$ in diameter were encapsulated by resorcinarene 6 and extracted to the aqueous interface. ${ }^{43}$ In each case, the encapsulated nanoparticles selforganized into hexagonally close-packed 2D arrays with good local order. Although the nanoparticles were presumably separated by two resorcinarene monolayers, close inspection of the TEM images revealed that the interparticle separation $\delta$ was on the order of $1 \mathrm{~nm}$ or less, which suggested that the surfactant chains were not interdigitated but compressed against the particle surfaces. This is in fact consonant with entropic steric repulsion, which depends on loss of chain mobility rather than direct physical hindrance.

A statistical analysis of interparticle spacings within welldefined domains of each $2 \mathrm{D}$ array demonstrated small but significant changes in $\delta$ (see Fig. 11). ${ }^{43}$ Despite the limited
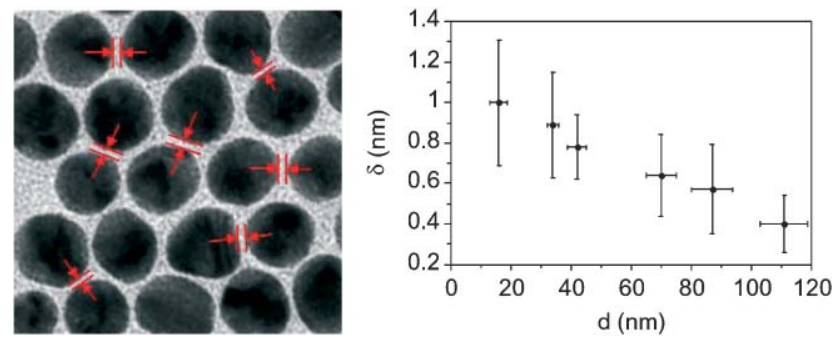

Fig. 11 Average particle diameters (d) vs. interparticle spacing parameters $(\delta)$ of Au nanoparticle arrays based on TEM image analysis at high digital resolution. ${ }^{43}$ Errors are equal to one standard deviation.

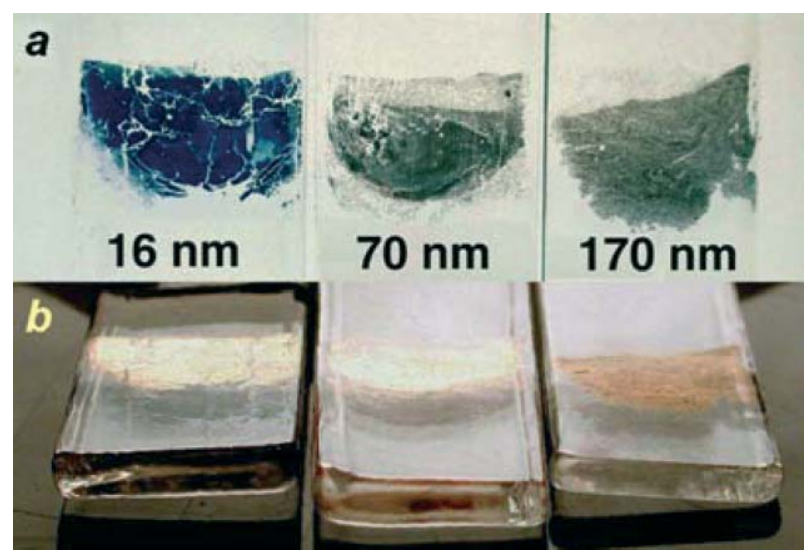

Fig. 12 (a) 16-, 70- and 170-nm Au particle arrays on smooth quartz substrates, photographed against a lit background. (b) Specular reflectance from $\mathrm{Au}$ nanoparticle arrays at a $60^{\circ}$ angle of incidence. ${ }^{47}$ Substrate width is approximately $1 \mathrm{~cm}$.

accuracy of these measurements, an inverse correlation between particle size and interparticle spacing could be established. The decremental changes in $\delta$ are likely due to the greater $\mathrm{vdW}$ attractive force as a function of particle size.

\section{Optical properties of gold nanoparticle arrays}

The dipolar plasmon resonances of the $\mathrm{Au}$ nanoparticles within the self-assembled 2D arrays were strongly coupled, resulting in a collective plasmon response. The Au nanoparticle arrays varied in hue from blue to a faint grey, and possessed several size-dependent optical properties ranging from visible to near-infrared (NIR) wavelengths (see Fig. 12). Extinction spectroscopy indicated a remarkable shifting and broadening of the plasmon band by hundreds of nanometers with increasing particle diameter (see Fig. 13). ${ }^{43,44}$ The sizedependent shift in the plasmon bands of the $2 \mathrm{D}$ arrays are much more pronounced than that of individual metal nanoparticles, because the electromagnetic couplings between

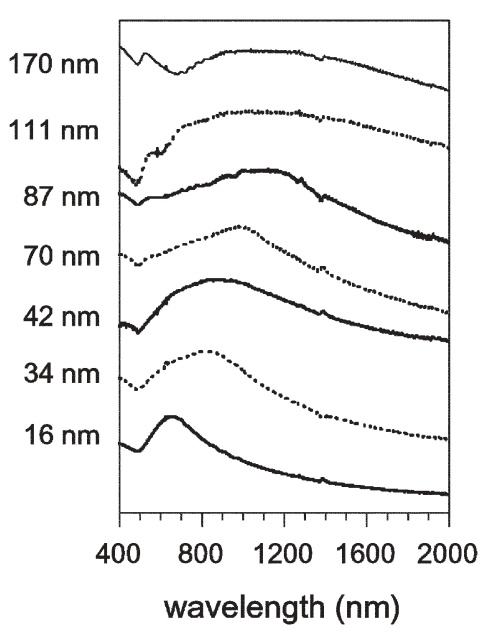

Fig. 13 Extinction spectra of Au nanoparticle arrays on quartz substrates. ${ }^{43}$ The collective plasmon bands are highly sensitive to incremental changes in array structure. 
particles are highly sensitive to structural changes. Interparticle spacing has an especially important influence on the collective properties of the nanoparticle arrays, as has been demonstrated in other optical studies of metal nanoparticle films. ${ }^{10,45,46}$

The specular reflectance of the 2D arrays was also dependent on periodic structure, with maximum reflectance of visible light observed for periodicities between 40 and $70 \mathrm{~nm}$ (see Fig. 12(b)). ${ }^{44,47}$ These variable reflectivities can be attributed to several effects: size-dependent optical absorption in the visible region for $2 \mathrm{D}$ arrays with periodicities below $40 \mathrm{~nm}$; angular-dependent attenuation in reflectance as a function of surface roughness; ${ }^{48}$ and size-dependent Mie scattering, which increase in significance for nanoparticles and metal surfaces with roughnesses of $40 \mathrm{~nm}$ or more. ${ }^{8,51}$

The 2D nanoparticle arrays exhibited size-dependent SERS activities, with signal intensities varying by two orders of magnitude under identical sampling conditions and surface coverages (see Fig. 14). ${ }^{44,47}$ SERS has attracted widespread interest as a spectroscopic mode of sensing: it can produce vibrational Raman spectra from just a few molecules in the presence of metal nanostructures, and has been shown to be capable of single-molecule detection. ${ }^{49}$ SERS is also compatible with water, and its unique combination of high sensitivity and high information content makes it an attractive method for detecting trace levels of analyte in a label-free manner. SERS is primarily derived from the electromagnetic fields generated by the surface plasmons in metal nanostructures, yet despite extensive efforts to elucidate the physical basis of the enhanced Raman signals, the rational design of SERS substrates with reproducible, ultrahigh sensitivity remains a challenging goal.

The structural and optical tunability of the Au nanoparticle 2D arrays provided an opportunity to better understand the relationship between SERS and the nanostructured substrate (see Fig. 15). Surface-averaged signal enhancement factors $(G)$ were calculated at three different excitation wavelengths by comparing the SERS signals from monolayers of $\mathbf{6}$ on the 2D

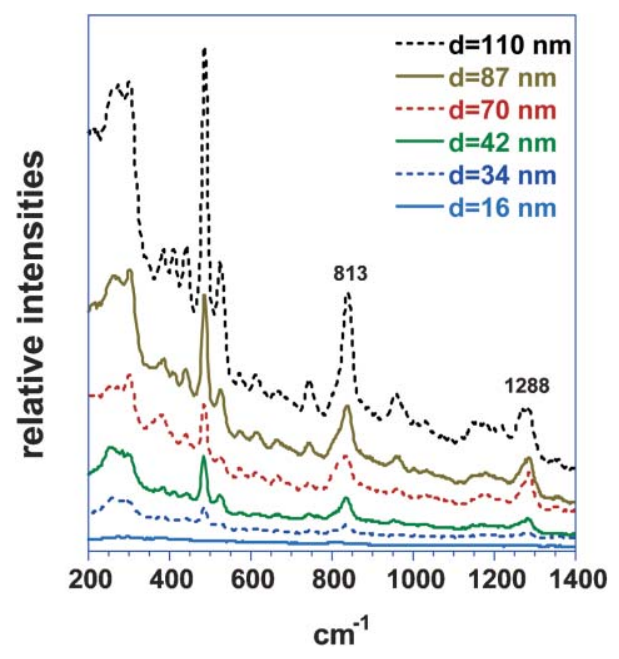

Fig. 14 SERS spectra of resorcinarene 6 from Au nanoparticle arrays, using an excitation wavelength of $785 \mathrm{~nm}\left(10 \mathrm{~mW}\right.$, area $=700 \mu \mathrm{m}^{2}$, exposure $=30 \mathrm{~s}) .{ }^{44}$ Spectra are shifted for clarity of presentation.
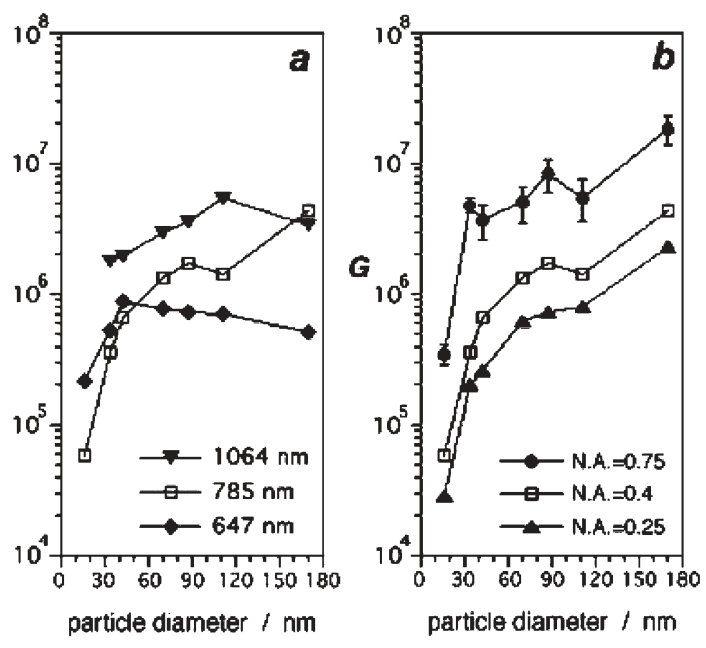

Fig. 15 SERS enhancement factors $(G)$ as a function of nanoparticle array structure, based on the integrated signal at $813 \mathrm{~cm}^{-1}$ from resorcinarene $6 .^{47}$ (a) $G$ values at excitation wavelengths of 647,785 and $1064 \mathrm{~nm}$; (b) $G$ values obtained at $785 \mathrm{~nm}$ using different solid angles, as determined by the numerical aperture (N.A.) of the collection objective.

arrays (normalized by surface density) with unenhanced Raman signals from resorcinarene films of known thickness. ${ }^{47}$ The SERS signal intensities were dependent on both excitation wavelength and array periodicity, which correlated with the size-dependent changes in plasmon resonance. Additional signal enhancement could be obtained by increasing the incident and collection solid angles to yield $G$ values in excess of $10^{7}$. The Au nanoparticle 2D arrays were also capable of detecting exogenous analytes by SERS, although their signals were not as strong as those from the surfactants themselves. Volatile organic compounds produced a detectable signal well before saturation adsorption, ${ }^{47}$ and removal of the resorcinarene layer by plasma treatment enabled the detection of analytes in aqueous solutions at micromolar levels. ${ }^{50}$

Theoretical studies on closely packed nanoparticles have indicated that the electromagnetic field enhancements responsible for SERS are highly localized between particle surfaces (see Fig. 16(a)). ${ }^{51,52}$ These local SERS enhancements can be many orders of magnitude greater than the surface-averaged enhancements, making self-assembly an appealing method to develop well-defined 'hot spots' for ultrasensitive SERS spectroscopy. On the other hand, restricted access to these highly active sites limits the usefulness of the close-packed 2D arrays for analyte detection.

Strategies to further improve analyte sensitivity have been identified by recent theoretical calculations on periodic $2 \mathrm{D}$ arrays of metal nanoparticles, which indicate that surfaceaveraged SERS can be optimized by adjusting the particle diameter-spacing ratio (see Fig. 16(b)). ${ }^{52}$ Resonant $G_{\mathrm{av}}$ values from optimized 2D arrays can be several orders of magnitude greater than those produced by disordered metal aggregates. In addition to field optimization, adjusting the spacing $\delta$ also increases the available sampling volume for analyte detection, a current limitation of the close-packed arrays. For example, the $G$ values of $100-\mathrm{nm}$ Au particle 2D arrays can be optimized for spacings between 3 and $10 \mathrm{~nm}$, whereas the spacings in the 

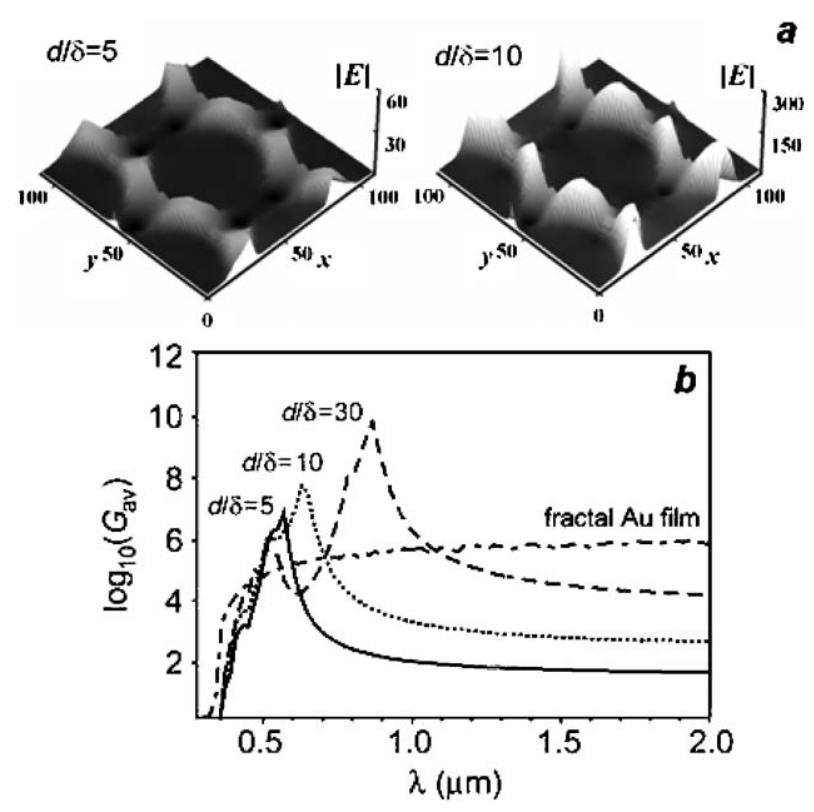

Fig. 16 (a) Electromagnetic field distributions within 2D arrays of $\mathrm{Au}$ nanoparticles with diameter-spacing ratios $(d / \delta)$ of 5 and 10 , excited by plane-polarized light $(\lambda=647 \mathrm{~nm}) .^{52}$ (b) Semilog plot of $G_{\text {av }}$ values from $\mathrm{Au}$ nanoparticle arrays with $d / \delta=5,10$ and 30 as a function of $\lambda$. The $G_{\text {av }}$ plot for a disordered (fractal) metal film is included for comparison.

resorcinarene-encapsulated nanoparticle arrays were measured to be less than $1 \mathrm{~nm}$.

\section{Self-assembly of cobalt nanoparticle rings}

Many magnetic properties have critical length scales in the 10 $100 \mathrm{~nm}$ range: single-domain particles with ferromagnetic behavior are typically found in this size regime, several of which have Curie points (the threshold between superparamagnetism and ferromagnetism) near ambient temperatures. ${ }^{53}$ With respect to self-assembly, ferromagnetic nanoparticles are subject to two types of attractive forces: nondirectional longe-range $\mathrm{vdW}$ forces which scale as an approximate function of $1 / D^{2}$, and medium-range dipolar attractions which increase as a function of $1 / D^{3}$, where $D$ is the distance between particle centers (i.e. $d+\delta$ ).

In order to extend the dispersion-enhancing properties of calixarenes toward magnetic nanoparticles, we used resorcinarene 1 to stabilize weakly ferromagnetic Co nanoparticles ( $d \sim 27 \mathrm{~nm}$ ) in nonpolar solvents such as toluene. Deposition of these stable dispersions onto carbon-coated TEM grids resulted in the unusual formation of bracelet-like nanoparticle rings, with the majority of these containing 5 to 10 particles (see Fig. 17). ${ }^{54,55}$ The median ring size could be reduced by diluting the dispersion prior to deposition, to produce rings less than $100 \mathrm{~nm}$ across. ${ }^{56}$ It is interesting to note that smaller Co nanoparticles $(d<15 \mathrm{~nm})$ generally did not participate in ring self-assembly, presumably due to their superparamagnetic nature at room temperature. Magnetic particles with strongly cooperative dipolar interactions are well known to assemble into chains, which provide the basis for field-induced changes in viscosity of ferrofluids. ${ }^{57}$ However, in situations where the

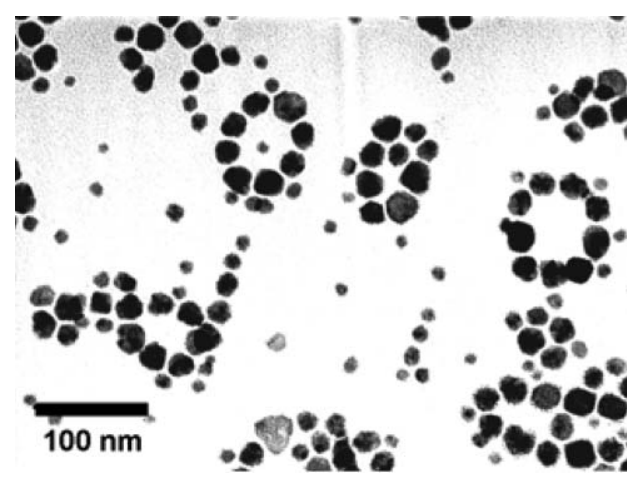

Fig. 17 TEM image (JEOL 2000 FX, $200 \mathrm{keV}$ ) of Co nanoparticle rings, deposited from a toluene dispersion containing resorcinarene $1 .^{54,55}$ Reprinted with kind permission of Springer Science and Business Media.

dipolar couplings are weak (again on the order of $k_{\mathrm{B}} T$ ), ring assembly is entropically favored over chains in the absence of applied magnetic fields.

Resorcinarene $\mathbf{1}$ was found to be essential for stabilizing the nanoparticle rings against "vdW collapse" as the particle dispersions were dried onto the TEM grids. ${ }^{55}$ Co nanoparticles deposited from toluene dispersions with minimal surfactant produced only densely packed particle films, indicating that nondirectional vdW forces were predominant. Increasing the amount of $\mathbf{1}$ produced a more viscous wetting layer, and effectively preserved the nanoparticle rings by immobilizing them at an early stage of the drying process. With respect to the mechanism of ring self-assembly, the role of magnetic dipolar interactions was confirmed by TEM analysis of samples prepared under the influence of a magnetic field, which induced a transition to oriented nanoparticle chains (see Fig. 18). However, removing the magnetic field resulted in the reformation of nanoparticle rings, demonstrating that their self-assembly was indeed thermodynamically favored over that of chains.

It is important to mention that several other mechanisms exist for depositing nanoparticles into ringlike assemblies down to submicron diameters. These include hole nucleation in unstable wetting layers, ${ }^{58}$ cell wall formation by RayleighBénard convection, ${ }^{59}$ and contact line pinning during surface dewetting. ${ }^{60}$ Such phenomena are kinetically driven by the

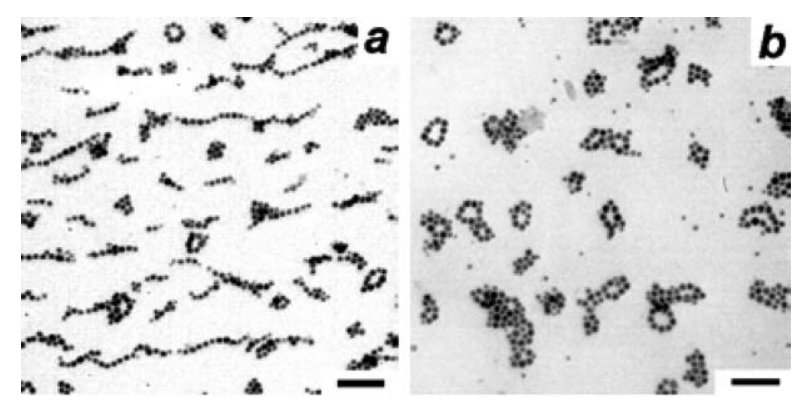

Fig. 18 (a) TEM image (Philips EM-400, $80 \mathrm{kV}$ ) of Co nanoparticle chains, deposited under the influence of a magnetic field $(B=225 \mathrm{G})$. (b) Reformation of nanoparticle rings, deposited 1 week after exposure to a strong magnetic field. ${ }^{55}$ Scale bar $=200 \mathrm{~nm}$. 


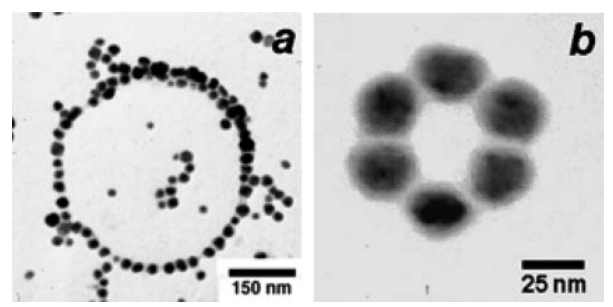

Fig. 19 (a) Co nanoparticle ring formed by a hole nucleation process, deposited from a $\mathrm{CH}_{2} \mathrm{Cl}_{2}$ dispersion containing resorcinarene $1 .{ }^{55}$ (b) Co nanoparticle ring formed by dipole-directed self assembly, deposited from a toluene dispersion.

rapid evaporation or recession of the wetting layer, and can be used to produce nanoparticle rings of high radial symmetry. In our case, deposition of resorcinarene-coated Co nanoparticles from a $\mathrm{CH}_{2} \mathrm{Cl}_{2}$ dispersion could produce submicron-sized rings via hole nucleation (see Fig. 19). ${ }^{55}$ However, a close inspection of such rings revealed numerous gaps between particles, whereas the nanoparticle rings formed by dipoledirected assembly were consistently in close contact. Like the $\mathrm{Au}$ nanoparticle 2D arrays of the previous section, the interparticle spacing has a strong influence over the coupling between magnetic nanoparticles, with a subsequent impact on their collective magnetic properties.

\section{Magnetic properties of cobalt nanoparticle rings: the chiral flux closure state}

Rings provide an ideal geometry for supporting a chiral magnetic state known as flux closure (FC), which has a net magnetostatic energy of zero and is polarized in either the clockwise $(\mathrm{CW})$ or counterclockwise $(\mathrm{CCW})$ direction (see Fig. 20). Magnetic rings with FC states are prime candidates as electronically addressable memory elements, because their polarizations can be reversed by passing an electrical current though their centers. Early magnetic memory devices were based on the FC states of millimeter-sized ferrite rings, prior to the advent of semiconductor-based data storage. ${ }^{61}$ Interest in magnetic rings has recently resurfaced as potential switching elements in devices based on magnetoresistive random-access memory (MRAM). ${ }^{62}$ Co rings as small as $100 \mathrm{~nm}$ across have been fabricated by electron-beam lithography, ${ }^{63}$ but further miniaturization is limited by the present resolution afforded by "top-down" methodologies.

Self-assembly offers an appealing alternative toward the preparation of magnetic nanorings below $100 \mathrm{~nm}$, and has the potential for integration with electronically conductive
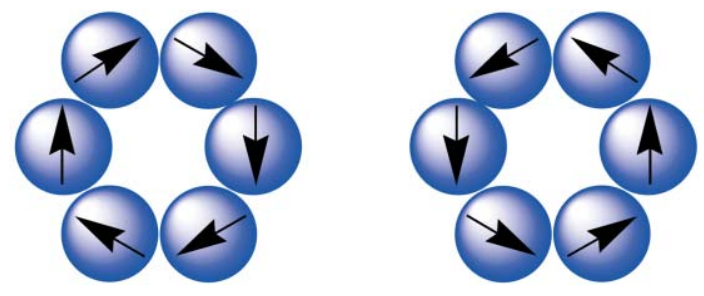

Fig. 20 Illustration of flux closure (FC) domains in cyclic ensembles of magnetic dipoles, in $\mathrm{CW}$ and $\mathrm{CCW}$ configurations. ${ }^{56}$

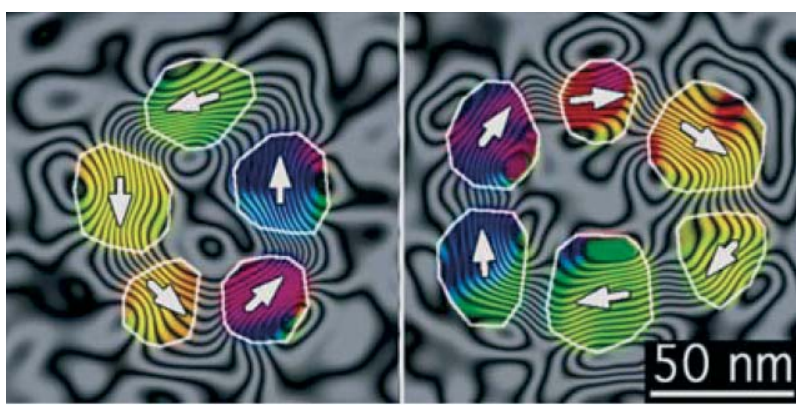

Fig. 21 Off-axis electron holography images of self-assembled Co nanoparticle rings, taken at $298 \mathrm{~K}$ using a Philips CM-300 equipped with a Lorentz lens $(300 \mathrm{keV})$. The polarization of the FC states are depicted using arrows and colors (red = right, yellow $=$ down, green $=$ left, blue $=$ up). The magnetic flux enclosed between adjacent contours is $h / 128 e$, and correlates inversely with the contour linewidths. ${ }^{56}$

nanostructures. In addition, the collective magnetic behavior of single-domain particles arranged in a circular geometry provides an interesting system which can be modeled by micromagnetics simulations. Although the physical size limit for FC stability in nanorings has not yet been determined, it is likely similar to the limit of ferromagnetic stability in singledomain particles, so there is considerable opportunity for further size reduction and densification.

To confirm that the self-assembled magnetic nanorings were indeed capable of supporting stable FC states, the Co nanoparticle rings were analyzed at $298 \mathrm{~K}$ by off-axis electron holography, in collaboration with Dunin-Borkowski (Dept. of Materials Science, University of Cambridge). ${ }^{56}$ Electron holography is a specialized TEM technique which enables inplane magnetic flux to be visualized with nanometer spatial resolution, and is one of the few methods which can be used to study magnetism in isolated nanostructures. ${ }^{64} \mathrm{~A}$ well-defined FC state could be imaged in every nanoparticle ring examined, despite irregularities in particle shape and deviations from radial symmetry (see Fig. 21). Furthermore, minimal magnetic flux was detected beyond the rings' outer perimeter or within their centers. These observations are consistent with a magnetostatic description of cyclic ensembles of single-domain particles.

Electron holography also records phase information which reveals the polarization of the FC states within the Co nanoparticle rings (see Fig. 21). A statistical sampling revealed a 50:50 (racemic) mixture of $\mathrm{CW}$ and $\mathrm{CCW}$ ground-state configurations, indicating the absence of polarization effects. The chirality of the FC states was stable at $298 \mathrm{~K}$, and was unaffected by external magnetic fields of up to several hundred Oe. This degree of coercivity suggests that even smaller nanoparticle rings can be expected to support chiral FC states at ambient temperatures.

\section{Conclusions}

The studies described in this feature article demonstrate that calixarenes, which have such a remarkable capacity for molecular encapsulation, are also proficient at encapsulating nanoparticles and enabling their self-assembly into nanostructures with well-defined properties. Whereas molecular encapsulation 
depends on supramolecular interactions between the capsule units as well as the guest molecule, nanoparticle encapsulation is mediated by chemisorptive interactions between the nanoparticle surface and multiple functional groups stationed around the calixarene rim. The calixarene's macrocyclic scaffold is also ideally suited for projecting chains at intermediate densities from the nanoparticle surface, to ensure that long-range vdW forces are effectively countered by entropic steric repulsion.

By combining capabilities in synthesis and self-assembly with theoretical efforts and high-resolution analytical methods, our understanding and control of nanoscale self-assembly can be applied toward the development and optimization of material function. For example, the characterization and modeling of the optical properties of the Au nanoparticle 2D arrays has revealed that their collective plasmon responses depend not only on particle size but also on interparticle spacing, suggesting directions for future efforts in synthesis and fabrication.

Conversely, the creation of new nanostructures may lead to unique insights in the structure-property relationships of nanoscale assemblies. In the case of the Co nanoparticle rings, we have recently discovered that their FC polarizations can be reversed by out-of-plane magnetic pulses. ${ }^{65}$ This novel switching mechanism has no known analogy at the macroscopic level yet can be reproduced by micromagnetic simulations, providing a strong incentive to further investigate and understand this phenomenon. Such efforts depend on a strong and sustainable synergy between disciplines, which has become increasingly important for converting scientific discoveries into useful new knowledge.

\section{Acknowledgements}

The author gratefully acknowledges past and present collaborators and research group members for their many contributions to this body of work. Financial support was provided by the National Science Foundation (BES-0086804, CHE-0243496, ECS-0210445), the National Institutes of Health (EB-001777-01), and the Defense Advanced Research Projects Agency (MDA972-03-0020) in association with the Birck Nanotechnology Center.

\section{References}

1 D. Jiles, Introduction to Magnetism and Magnetic Materials, Chapman and Hall, New York, 1991.

2 (a) C. P. Collier, T. Vossmeyer and J. R. Heath, Annu. Rev. Phys. Chem., 1998, 49, 371; (b) M. P. Pileni, J. Phys. Chem. B, 2001, 105, 3358.

3 A. G. S. Högberg, J. Am. Chem. Soc., 1980, 102, 6046.

4 D. J. Cram, Nature, 1992, 356, 29.

5 Y. Aoyama, Y. Tanaka, H. Toi and H. Ogoshi, J. Am. Chem. Soc., 1988, 110, 634.

6 P. Timmerman, W. Verboom and D. N. Reinhoudt, Tetrahedron, 1996, 52, 2663.

7 For functionalization of $\mathrm{Au}$ nanoparticles by perthiolated cyclodextrins, see: J. Liu, S. Mendoza, E. Román, M. J. Lynn, R. Xu and A. E. Kaifer, J. Am. Chem. Soc., 1999, 121, 4304.

8 (a) C. P. Collier, T. Vossmeyer and J. R. Heath, Annu. Rev. Phys. Chem., 1998, 49, 371; (b) M. P. Pileni, J. Phys. Chem. B, 2001, 105, 3358.

9 P. L. McEuen, Science, 1997, 287, 1729.
10 C. P. Collier, R. J. Saykally, J. J. Shiang, S. E. Henrichs and J. R. Heath, Science, 1997, 277, 1978.

11 C. T. Black, C. B. Murray, R. L. Sandstrom and S. Sun, Science, 2000, 290, 1131.

12 (a) A. N. Shipway, E. Katz and I. Willner, ChemPhysChem, 2000, 1, 18; (b) J. Zhang, Z.-L. Wang, J. Liu, S. Chen and G.-Y. Liu, Self-Assembled Nanostructures, ed. D. J. Lockwood, Springer, New York, 2003; (c) Nanoparticles: Scaffolds and Building Blocks, ed. V. M. Rotello, Kluwer, New York, 2004.

13 (a) F. Kim, S. Kwan, J. Akana and P. Yang, J. Am. Chem. Soc., 2001, 123, 4360; (b) L. S. Li, J. Walda, L. Manna and A. P. Alivisatos, Nano Lett., 2002, 2, 557; (c) L. S. Li and A. P. Alivisatos, Adv. Mater., 2003, 15, 408; (d) N. R. Jana, Angew. Chem., Int. Ed., 2004, 43, 1536.

14 Y. Huang, X. Duan, Q. Wei and C. M. Lieber, Science, 2001, 291, 630.

15 (a) L. Cusack, R. Rizza, A. Gorelov and D. Fitzmaurice, Angew. Chem., Int. Ed. Engl., 1997, 36, 848; (b) J. J. Storhoff and C. A. Mirkin, Chem. Rev., 1999, 99, 1849; (c) J. Simard, C. Briggs, A. K. Boal and V. M. Rotello, Chem. Commun., 2000, 1943; (d) B. L. Frankamp, O. Uzun, F. Ilhan, A. K. Boal and V. M. Rotello, J. Am. Chem. Soc., 2002, 124, 892.

16 M. Kanehara, Y. Oumi, T. Sano and T. Teranishi, J. Am. Chem. Soc., 2003, 125, 8708.

17 (a) C. J. Kiely, J. Fink, M. Brust, D. Bethell and D. J. Schiffrin, Nature, 1998, 396, 444; (b) B. Cui, B. Lin and S. A. Rice, J. Chem. Phys., 2003, 119, 2386; (c) F. X. Redl, K.-S. Cho, C. B. Murray and S. O'Brien, Nature, 2003, 423, 968 .

18 (a) J. R. Heath, C. M. Knobler and D. V. Leff, J. Phys. Chem. B, 1997, 101, 189; (b) W. M. Gelbart, R. P. Sear, J. R. Heath and S. Chaney, Faraday Discuss., 1999, 112, 299.

19 L. Motte and M. P. Pileni, J. Phys. Chem. B, 1998, 102, 4104.

20 Selected examples: (a) M. M. Maye, W. Zheng, F. L. Leibowitz, N. K. Ly and C. J. Zhong, Langmuir, 2000, 16, 490; (b) V. F. Puntes, K. M. Krishnan and A. P. Alivisatos, Appl. Phys. Lett., 2001, 78, 2187; (c) L. O. Brown and J. E. Hutchison, J. Phys. Chem. B, 2001, 105, 8911 .

21 X. M. Lin, H. M. Jaeger, C. M. Sorensen and K. J. Klabunde, J. Phys. Chem. B, 2001, 105, 3353.

22 Selected examples: (a) B. O. Dabbousi, C. B. Murray, M. F. Rubner and M. G. Bawendi, Chem. Mater., 1994, 6, 216; (b) F. C. Meldrum, N. A. Kotov and J. H. Fendler, Chem. Mater., 1995, 7, 1112; (c) J. J. Brown, J. A. Porter, C. P. Daghlian and U. J. Gibson, Langmuir, 2001, 17, 7966.

23 Selected examples: (a) C. B. Murray, C. R. Kagan and M. G. Bawendi, Science, 1995, 270, 1335; (b) P. J. Thomas, G. U. Kulkarni and C. N. R. Rao, J. Phys. Chem. B, 2001, 105, 2515; (c) D. V. Talapin, E. V. Shevchenko, A. Kornowski, N. Gaponik, M. Haase, A. L. Rogach and H. Weller, Adv. Mater., 2001, 13, 1868; (d) S. I. Stoeva, B. L. V. Prasad, S. Uma, P. K. Stoimenov, V. Zaikovski, C. M. Sorensen and K. J. Klabunde, J. Phys. Chem. B, 2003, 107, 7441 .

24 V. Santhanam and R. P. Andres, Nano Lett., 2004, 4, 41.

25 (a) U. Kreibig and M. Vollmer, Optical Properties of Metal Clusters, Springer, New York, 1995; (b) J. Yguerabide and E. E. Yguerabide, Anal. Biochem., 1998, 262, 137; (c) A. Wei, in Plasmonic Nanomaterials: Enhanced Optical Properties From Metal Nanoparticles and their Ensembles, ed. V. M. Rotello, Kluwer, New York, 2004

26 D. Jiles, Introduction to Magnetism and Magnetic Materials, Chapman and Hall, New York, 1991.

27 (a) S. Alexander, J. Phys., 1977, 38, 977; (b) P.-G. DeGennes, Macromolecules, 1980, 13, 1069.

28 L. H. Dubois and R. G. Nuzzo, Annu. Rev. Phys. Chem., 1992, 43, 437.

29 (a) L. R. MacGillivray and J. L. Atwood, Nature, 1997, 389, 469; (b) J. L. Atwood, L. J. Barbour and A. Jerga, Chem. Commun., 2001, 2376; (c) R. M. McKinlay, G. W. V. Cave and J. L. Atwood, Proc. Natl. Acad. Sci. USA, 2005, 102, 5944.

30 O. D. Fox, M. G. B. Drew and P. D. Beer, Angew. Chem., Int. Ed., 2000, 39, 136.

31 (a) A. Shivanyuk and J. Rebek, Proc. Natl. Acad. Sci. USA, 2001, 98, 7662; (b) M. Yamanaka, A. Shivanyuk and J. Rebek, J. Am. Chem. Soc., 2004, 126, 2939; (c) L. Avram and Y. Cohen, J. Am. Chem. Soc., 2004, 126, 11556. 
32 (a) A. M. Nechifor, A. P. Philipse, F. de Jong, J. P. M. van Duynhoven, R. J. M. Egberink and D. N. Reinhoudt, Langmuir, 1996, 12, 3844; (b) X.-M. Li, M. R. de Jong, K. Inoue, S. Shinkai, J. Huskens and D. N. Reinhoudt, J. Mater. Chem., 2001, 11, 1919 .

33 (a) A. V. Nabok, T. Richardson, F. Davis and C. J. M. Stirling, Langmuir, 1997, 13, 3198; (b) J. Liu, W. Ong and A. E. Kaifer Langmuir, 2002, 18, 5981.

34 M. Ueda, N. Fukushima, K. Kudo and K. Ichimura, J. Mater. Chem., 1997, 7, 641 .

35 K. B. Stavens, S. V. Pusztay, S. Zou, R. P. Andres and A. Wei, Langmuir, 1999, 15, 8337.

36 S. V. Pusztay, A. Wei, K. B. Stavens and R. P. Andres, Supramol. Chem., 2002, 14, 291.

37 R. Balasubramanian and A. Wei, unpublished results.

38 (a) A. Wei, K. B. Stavens, S. V. Pusztay and R. P. Andres, MRS Symp. Proc. Ser., 1999, 581, 59; (b) R. Balasubramanian, J. Xu, B. Kim, B. Sadtler and A. Wei, J. Dispersion Sci. Tech., 2001, 22, 485 .

39 R. Balasubramanian, B. Kim, S. L. Tripp, X. Wang, M. Lieberman and A. Wei, Langmuir, 2002, 18, 3676.

40 Y. Zhao, W. Pérez-Segarra, Q. Shi and A. Wei, J. Am. Chem. Soc., 2005, 127, 7328

41 B. Kim, M. A. Carignano, S. L. Tripp and A. Wei, Langmuir, 2004, 20, 9360 .

42 B. Kim, R. Balasubramanian, W. Pérez-Segarra, A. Wei, B. Decker and J. Mattay, Supramol. Chem., 2005, 17, 173.

43 B. Kim, S. L. Tripp and A. Wei, J. Am. Chem. Soc., 2001, 123, 7955.

44 B. Kim, S. L. Tripp and A. Wei, MRS Symp. Proc., 2001, 676, Y.6.1.

45 J. J. Shiang, J. R. Heath, C. P. Collier and R. J. Saykally, J. Phys. Chem. B, 1998, 102, 3425.

46 T. Ung, L. M. Liz-Marzán and P. Mulvaney, J. Chem. Phys. B, 2001, 105, 3441.

47 A. Wei, B. Kim, B. Sadtler and S. L. Tripp, ChemPhysChem, 2001, 2, 743 .

48 H. Raether, Surface Plasmons on Smooth and Rough Surfaces and on Gratings, Springer, New York, 1988.
49 K. Kneipp, H. Kneipp, I. Itzkan, R. R. Dasari and M. S. Feld, Chem. Rev., 1999, 99, 2957.

50 B. Kim and A. Wei, unpublished results.

51 H. Xu, J. Aizpurua, M. Käll and P. Apell, Phys. Rev. E, 2000, 62, 4318 .

52 D. A. Genov, A. K. Sarychev, V. M. Shalaev and A. Wei, Nano Lett., 2004, 4, 153.

53 (a) N. Spaldin, Magnetic Materials: Fundamental and Device Applications, Cambridge University Press, Cambridge, 2003.

54 A. Wei, B. Kim, S. V. Pusztay, S. L. Tripp and R. Balasubramanian, J. Inclusion Phenom. Macrocycl. Chem. 2001, 41, 83 .

55 S. L. Tripp, S. V. Pusztay, A. E. Ribbe and A. Wei, J. Am. Chem. Soc., 2002, 124, 7914

56 S. L. Tripp, R. E. Dunin-Borkowski and A. Wei, Angew. Chem. Int. Ed., 2003, 42, 5591.

57 R. E. Rosensweig, Ferrohydrodynamics, Cambridge University Press, Cambridge, 1985.

58 (a) P. C. Ohara, J. R. Heath and W. M. Gelbart, Angew. Chem., Int. Ed. Engl., 1997, 36, 1078; (b) P. C. Ohara and W. M. Gelbart, Langmuir, 1998, 14, 3418; (c) T. Vossmeyer, S.-W. Chung, W. M. Gelbart and J. R. Heath, Adv. Mater., 1998, 10, 351.

59 M. Maillard, L. Motte, A. T. Ngo and M. P. Pileni, J. Phys. Chem $B, 2000,104,11871$

60 (a) R. D. Deegan, O. Bakajin, T. F. Dupont, G. Huber, S. R. Nagel and T. A. Witten, Nature, 1997, 389, 827; (b) R. D. Deegan, Phys. Rev. E, 2000, 61, 475 .

61 L. A. Russell, R. M. Whalen and H. O. Leilich, IEEE Trans. Magn., 1968, 4, 134.

62 J.-G. Zhu, Y. Zheng and G. A. Prinz, J. Appl. Phys., 2000, 87, 6668.

63 L. J. Heyderman, C. David, M. Kläui, C. A. F. Vaz, J. Rothman and J. A. C. Bland, J. Appl. Phys., 2003, 93, 10011.

64 (a) R. E. Dunin-Borkowski, M. R. McCartney, B. Kardynal, S. S. P. Parkin, M. R. Scheinfein and D. J. Smith, J. Microsc., 2000, 200, 187; (b) R. E. Dunin-Borkowski, T. Kasama, A. Wei, S. L. Tripp, M. J. Hytch, E. Snoeck, R. J. Harrison and A. Putnis, Microsc. Res. Tech., 2004, 64, 390.

65 T. Kasama, R. E. Dunin-Borkowski, M. R. Scheinfein, S. L. Tripp and $\mathrm{A}$. Wei, unpublished results. 